\title{
Fatores de estresse na atividade de médicos em João Pessoa (PB, Brasil)
}

\author{
Cidalia de Lourdes Moura Santos, ${ }^{\mathrm{a}, *}$, Celso Luiz Pereira Rodrigues, \\ Luiz Bueno da Silvac, Hanne Alves Bakked, \\ Alexandre Santos de Moura Leite ${ }^{\mathrm{e}}$, Maria Marta de Araújo Leal ${ }^{\mathrm{f}}$ \\ a,*cimoura@hs24.com.br, UFPB, Brasil \\ bcelsoluiz@ct.ufpb.br, UFPB, Brasil \\ cbueno@ct.ufpb.br, UFPB, Brasil \\ `h_bakke@yahoo.com.br, UFPB, Brasil \\ easmleite@gmail.com, UFPB, Brasil \\ fmmartaaraujo@gmail.com, UFPB, Brasil
}

\begin{abstract}
Resumo
0 objetivo deste trabalho é verificar que fatores têm interferência direta no estresse de profissionais médicos em João Pessoa (PB) baseado nas variáveis de uma adaptação do Inventário de Estresse em Enfermeiros (IEE) proposto por Stacciarini and Trocoli (2000). Os dados foram coletados por meio de um questionário semiestruturado, composto por perguntas de identificação do colaborador seguidas de 44 questões de múltipla escolha, aplicado a 391 médicos que trabalhavam em hospitais. As variáveis não métricas foram submetidas à análise fatorial, que indicou a presença de oito fatores para o estresse entre esses profissionais. Nesses fatores, é possível apontar variáveis que apresentam maior impacto, a saber: relacionamento com a equipe médica, relacionamento com os colegas médicos, trabalhar em equipe, relacionamento com a chefia, trabalhar em instalações físicas inadequadas, falta de material necessário ao trabalho e trabalhar em horário noturno. Esses resultados podem contribuir para o gerenciamento do estresse entre médicos.
\end{abstract}

Palavras-chaves

Estressores. Médicos. Hospitais.

\section{Introdução}

0 estresse representa um recurso biológico existente no organismo humano para preservação da saúde, em que um conjunto de reações e respostas ocorre para manter a integridade do organismo e preparar o corpo para enfrentar situações que requerem adaptação. Trata-se de um mecanismo químico e hormonal de defesa frente às ameaças do cotidiano. Segundo Oliveira e Kilimnik (2007), quando acompanhado de reações fisiológicas e de entusiasmo, vitalidade, otimismo e força física possui um aspecto positivo, sendo denominado de eustresse. Mas pode também apresentar um aspecto patológico predispondo o indivíduo ao cansaço, irritabilidade e doenças, sendo, nesse caso, chamado de distresse.
Agentes estressores estão presentes em qualquer tipo de atividade e não se deve esperar que um trabalho não produza estresse. Ele é necessário à execução de uma tarefa de forma eficiente. Para Dejours (1992), o trabalho é favorável ao trabalhador quando oferece uma possibilidade de concretizar suas aspirações, ideias e desejos, quando é livremente escolhido e quando a organização do trabalho é flexivel, para que ele possa adaptá-la a seus desejos, ao seu corpo e ao seu estado emocional. Todavia, muitas vezes a atividade de trabalho coloca o homem numa situação conflituosa. Se por um lado ela garante os meios de vida do ser humano, por outro a presença de demandas do ambiente, 
que exigem mais do que a estrutura psíquica do indivíduo suporta, pode gerar patologias e a diminuição da eficiência do homem, contribuindo para o surgimento do estresse de sobrecarga e o adoecimento.

Profissionais da área de saúde, incluindo médicos, têm diminuído a capacidade de produção, realizando atividades com menor precisão, aumentado o absenteísmo, adoecido com maior frequência, trabalhado tensos e cansados. E estão ansiosos e depressivos, com atenção dispersa, desmotivados e com baixa realização pessoal devido ao alto grau de estresse em suas atividades (BOLLER, 2003). Visto que a satisfação pessoal e a produtividade influem no equilíbrio entre o indivíduo e a organização, as empresas deveriam buscar gerar o desenvolvimento humano, potencializar as capacidades, garantir o equilíbrio entre as esferas profissional e individual de forma a preencher os requisitos de bem-estar, segurança e saúde (LIMONGI-FRANÇA, 2008). Segundo Pacheco Junior et al. (2000), a maior problemática relativa à segurança, medicina e higiene do trabalho é viabilizar a concepção estratégica de sua atuação, já que é um processo contínuo, necessitando de gestão própria e integrada à gestão global da organização.

Uma forma de contribuir para esse controle é através de pesquisas que retratem o estresse em médicos, pois o conhecimento dessas variáveis pode apoiar tomadas de decisões gerenciais, fornecer subsídios para fortalecer a implementação de projetos eficazes de segurança e saúde do trabalho, além de prevenir e/ou poder controlar os riscos promovidos por essas variáveis, contribuindo de maneira substantiva na redução de acidentes e doenças nesses profissionais. Dessa forma, com a realização deste trabalho, objetiva-se verificar quais fatores têm interferência direta no estresse de profissionais médicos da cidade de João Pessoa (PB), baseado na adaptação de algumas variáveis utilizadas no Inventário de Estresse em Enfermeiros (IEE) proposto por Stacciarini e Trocoli (2000).

\section{Estresse em médicos}

Hans Selye, médico endocrinologista, foi o primeiro cientista a utilizar o termo stress na área da saúde (SPIELBERGER, 1972). Em 1936, ele definiu stress como "o resultado inespecífico de qualquer demanda sobre o corpo, seja de efeito mental ou somático" (SPIELBERGER, 1972), e estressor "como todo agente ou demanda que provoca a reação de estresse, seja de natureza física, mental ou emocional" (SPIELBERGER, 1972). Em seus estudos, Selye observou que o estresse produzia reações de defesa e adaptação frente ao agente estressor e descreveu a sindrome geral de adaptação (SAG), caracterizada por três fases ou estágios: fase de alarme, fase de resistência e fase de exaustão.

A primeira fase, a de alarme, é uma posição de alerta do organismo com uma excitação de agressão ou de fuga ao estressor, e é reconhecida como uma situação de reação saudável, pois possibilita o retorno à situação de equilíbrio (CAMELO; ANGERAMI, 2004). A fase de resistência, ainda segundo os autores, consiste na persistência da fase de alerta com alterações nos parâmetros de normalidade e concentração da reação interna em um determinado órgão-alvo. Há nessa fase manifestação de sintomas da esfera psicossocial, como ansiedade, medo, isolamento social, roer unhas, oscilação do apetite, impotência sexual e outros. Na terceira fase, a de exaustão ou esgotamento, o organismo encontra-se extenuado pelo excesso de atividades e pelo alto consumo de energia. Essa fase é caracterizada por reações de sobrecarga dos canais fisiológicos, falhas dos mecanismos de adaptação, presença de estímulos permanentes e excessivos ocasionando, então, a falência do órgão-alvo. Segundo limongiFrança (2008), quando há falhas nos mecanismos adaptativos, desenvolvem-se doenças de adaptação, que são resultado de um conjunto equilibrado de defesa e submissão. Assim, alguns distúrbios podem ser ocasionados pelo excesso de hostilidade; outros por reações físicas de submissão excessivas.

A reação frente a um estressor envolve uma série de variáveis, dentre elas a intensidade com que ele acontece, as experiências passadas, o nível de ajustamento da personalidade e a interação entre o indivíduo e o ambiente. Os agentes estressores em um ambiente de trabalho são absorvidos de modo diferente por cada pessoa exposta. Sabe-se que o potencial estressor das mudanças fisiológicas advindas com o estresse é muito maior para indivíduos que ocupam determinados cargos, em que o grau de responsabilidade e poder de decisão desempenham importantes papéis dentro de um mesmo campo profissional. 0 convivio frequente com essas condições acarreta prejuízos na realização das atividades diárias, dificultando o relacionamento entre os trabalhadores, prejudicando a capacidade de atenção e concentração, causando lapsos de memória, aumentando o absenteísmo e os acidentes de trabalho, diminuindo assim a produtividade (BAUK, 1985). Pode-se citar, também, o desgaste emocional devido às relações com o trabalho, as quais são fatores significativos na determinação de transtornos relacionados ao estresse. É o caso das depressões, ansiedade patológica, pânico, fobias e doenças psicossomáticas, dentre outras (BALlONE, 2002). 
Os profissionais médicos estão sujeitos a estresse de várias naturezas e níveis. Algumas das características inerentes à tarefa médica definem, isoladamente ou em seu conjunto, um ambiente profissional formado pelos intensos estímulos emocionais que acompanham o adoecer (NOGUEIRA-MARTINS, 1991; CANDEIAS; ABUJAMRA; LIM, 1988): o contato íntimo e frequente com a dor e o sofrimento; tratar pacientes difíceis - queixosos, rebeldes e não aderentes ao tratamento, hostis, reivindicadores, autodestrutivos, cronicamente deprimidos -; o atendimento de pacientes terminais; lidar com a intimidade corporal e emocional; e lidar com incertezas e limitações do conhecimento médico e do sistema assistencial que se contrapõem às demandas e expectativas dos pacientes e familiares que desejam certezas e garantias.

Frasquilho (2005) aponta ainda como fatores de estresse na prática médica a sua formação universitária, a representação social, as condições de trabalho, a tarefa e a carga físico- mental, a organização do trabalho e o próprio médico. Em um estudo realizado sobre as condições de trabalho e de saúde dos médicos em Salvador, Nascimento Sobrinho et al. (2006) apontam para um cenário desfavorável para os médicos devido à sobrecarga de trabalho, especialmente em atividades de plantão; condições de trabalho precárias, regidas preferencialmente pela remuneração por procedimento, em especial no setor privado; baixa remuneração e elevada frequência de queixas físicas e psíquicas. lsso pode refletir na sociedade, através da baixa qualidade do atendimento prestado aos usuários dos serviços de saúde. Esses resultados explicam o descontentamento dos médicos e da sociedade em geral com os serviços de saúde e com a atenção médica.

Em uma pesquisa com 61 profissionais médicos, Lentine, Sonoda e Biazin (2003) identificaram que $77 \%$ consideraram o seu trabalho como estressante. Outro estudo realizado com 4.351 médicos em Goiás (SILVA, 2001) identificou um percentual de 83,8 profissionais se referiram a uma sensação de desgaste com o desempenho de sua atividade.

Oliveira e Kilimnik (2007), em um estudo com médicos ultrassonografistas de duas instituições em Minas Gerais, identificaram nos participantes os sintomas atribuíveis ao estresse tais como: nervosismo, ansiedade, irritabilidade, fadiga e dor muscular em 75\% dos médicos, e angústia em 65\%.

Além disso, a síndrome do esgotamento profissional/estafa/burn out tem sido descrita principalmente em profissionais que trabalham na prestação de cuidados a pessoas doentes, grupos sociais carentes e crianças, como enfermeiros, médicos, assistentes sociais, psicólogos e executivos (FREUDENBERG, 1974 apud MENDES, 1995; FRANÇA; RODRIGUES, 1999). Trata-se de uma condição caracterizada por: sintomas somáticos como exaustão, fadiga, cefaleias, distúrbios gastrintestinais, insônia e dispneia; e sintomas psíquicos como humor depressivo, irritabilidade, ansiedade, rigidez, negativismo, ceticismo e desinteresse. Um profissional que está burning-out tende a criticar tudo e todos que o cercam, tem pouca energia para as diferentes solicitações de seu trabalho, desenvolve frieza e indiferença para com as necessidades e o sofrimento dos outros, tem sentimentos de decepção e frustração e comprometimento da autoestima, evita pacientes, realiza consultas rápidas, evita contato visual e usa rótulos depreciativos (RODRIGUES, 1998; RAMOS-CERQUEIRA; LIMA, 2002).

Os agentes estressores podem se tornar mais presentes de acordo com a especialidade médica. Cabana et al. (2007), em um hospital público em Pernambuco, observaram que os médicos que trabalhavam no setor de emergência apresentavam um cotidiano com condições piores de trabalho quando comparados aos das enfermarias e Unidades de Terapia Intensiva (UTI). Quando questionados sobre a sensação de sobrecarga de trabalho, aqueles que atuavam no setor de emergência sentiam-se mais sobrecarregados, seguidos dos profissionais da UTl e das enfermarias. Lourenço (1998) chama atenção para o estresse gerado entre médicos-cirurgiões e anestesiologistas durante os procedimentos cirúrgicos ou até mesmo antes de iniciar uma cirurgia, principalmente em caso de urgências, mesmo numa equipe com anos de experiência. Durante o ato cirúrgico podem ocorrer picos de estresse, levando à desorganização da mente e do comportamento do ser profissional envolvido.

0 suicídio entre os profissionais médicos também é relatado na literatura. Um estudo realizado com esses profissionais na Inglaterra e no País de Gales revelou que os suicídios representam 38\% das mortes precoces em médicos, sendo quatro vezes superior entre médicas que na população em geral (HAWTON et al., 2001). Outra pesquisa demonstrou taxas de suicídio mais altas que a população geral, sendo a de médicas substancialmente mais elevada que a de médicos (SCHERNHAMMER; COLDITZ, 2004). Uma revisão de literatura realizada por Meleiro (1998) aponta como fatores de risco o conhecimento farmacológico que o médico possui, tornando qualquer tentativa de suicídio altamente letal, e o uso abusivo de drogas e álcool nessa 
população. Essas pesquisas chamam atenção para a necessidade de gerenciar o estresse e os problemas de saúde mental nesses profissionais de forma mais efetiva.

Segundo Iversen, Rushforth e Forrest (2009), o sistema pode auxiliar na prevenção de estresse e na saúde mental dos médicos fornecendo consultas confidenciais, treinamentos, psicoterapia e tratamento para problemas relacionados a álcool e drogas. Além disso, esses profissionais devem ter acesso a consultas, admissões e internações, caso seja necessário, fora de seu ambiente de trabalho.

Sabe-se que há no trabalho médico condições desencadeadoras de estresse e ansiedade e que elas interferem na produtividade e na qualidade do serviço prestado. Todavia, as repercussões sobre a saúde desses profissionais, expostos a fatores estressantes e ansiogênicos, ainda são pouco conhecidas. Por isso, a prevenção de doenças relacionadas ao estresse é atualmente o foco de muitas pesquisas. No entanto, poucas lidam com as variáveis estressoras e o impacto destas para 0 surgimento de estresse entre esses profissionais.

\section{Procedimentos metodológicos}

Trata-se de uma pesquisa exploratória que estuda os fatores relacionados ao estresse em médicos da cidade de João Pessoa (PB). Os dados foram coletados por meio de um questionário semiestruturado, composto por perguntas de identificação do colaborador seguido de 44 questões de múltipla escolha adaptadas do Inventário de Estresse em Enfermeiros - IEE (STACCIARINI; TROCOLl, 2000) e utilizadas para avaliação do estresse entre profissionais médicos. As questões fechadas apresentavam as seguintes alternativas de respostas: nunca, raramente, algumas vezes, muitas vezes e sempre, às quais foram atribuídos os valores $1,2,3,4$ e 5 , respectivamente.

A amostra foi composta por 391 médicos, independente da especialidade, que trabalhavam em hospitais da cidade de João Pessoa (PB) e aceitaram participar do estudo. Profissionais que exerciam suas atividades exclusivamente em clínicas, postos de saúde e centros de imagem foram excluídos do estudo.

Os dados relativos à identificação dos colaboradores foram analisados através da estatística descritiva para se obter uma visão global da variação desses valores, organizando e descrevendo os dados por meio de tabelas, gráficos e medidas de posição e de dispersão. A avaliação do comportamento das 44 variáveis não métricas simultaneamente foi realizada aplicando-se a técnica multivariada análise fatorial (AF). 0 objetivo principal desse tipo de análise é descrever as relações de covariância entre as variáveis em alguns fatores ocultos e inobserváveis. Sua utilização supõe que as variáveis podem ser agrupadas de acordo com suas correlações. Assim, obtém-se como resultado grupos com todas as variáveis altamente correlacionadas entre si, mas com baixas correlações com variáveis de um grupo diferente. Ao resumir os dados, a $\mathrm{AF}$ capta as dimensões latentes que representam o conjunto de dados em um número menor de conceitos do que as variáveis individuais originais (HAIR JUNIOR et al., 2005).

A análise dos resultados das variáveis não métricas seguiu os seguintes passos:

\subsection{Análise da confiabilidade dos dados}

Realizada através do modelo Alfa de Cronbach (CORRAR; PAULO; DIAS FILHO, 2007; HAIR JUNIOR et al., 2005), analisando-se a consistência interna dos indicadores individuais da escala utilizada no instrumento de pesquisa. Adotou-se como limite inferior de aceitabilidade o intervalo de 0,60 a 0,70 , ou seja, 60 a $70 \%$ representam o impacto real mínimo das variáveis. Esse Alfa é calculado através da Equação 1:

$\alpha=\frac{k \cdot \bar{c}}{\bar{v}+(k-1) \cdot \bar{c}}$

onde $k$ é o número de variáveis consideradas; $\bar{v}$ é a média das variâncias e $\bar{c}$ a média de todas as covariâncias.

\subsection{Teste de esfericidade de Bartlett}

Analisado através da estatística qui-quadrado ao nível de 0,05, examinou-se a hipótese de que as variáveis no estudo não sejam correlacionadas na população.

\subsection{Medida de adequacidade da amostra de Kaiser-Meyer-Olkin (KMO)}

Trata-se de um teste de adequacidade à análise fatorial. Valores altos (entre 0,5 e 1,0) indicam que esse método é apropriado.

Com a avaliação da adequacidade, analisaram-se as correlações parciais para verificar se as variáveis podem ou não ser explicadas por seus respectivos fatores. 0 número de fatores foi determinado utilizando-se o critério do autovalor 
e a porcentagem da variância explicada. A rotação utilizada neste estudo foi a Varimax. Cada fator foi submetido ao teste de confiabilidade, de acordo com a Equação 1, para analisar a estrutura interna de cada um, excluindo-se o fator com impacto abaixo de $60 \%$. A composição dos fatores, cujos valores de confiabilidade foram acima desse valor, foi apresentada listando suas respectivas influências e/ou importâncias.

Visto que a força do método utilizado (AF) está associada à proporção entre o número de variáveis a serem analisadas e o tamanho da amostra, buscou-se atingir uma proporção entre cinco e dez questionários respondidos para cada variável, motivo pelo qual foram totalizados 391 questionários. As questões não respondidas ou rasuradas foram substituídas pelos valores médios da referida variável, visto que um valor em branco descarta todas as respostas de um questionário, com consequente rejeição de vários formulários.

Todos os dados foram analisados com o software SPSS (v. 13) e receberam tratamento estatístico no Laboratório de Métodos Quantitativos Aplicados (LabMEQA) do Departamento de Engenharia de Produção da Universidade Federal da Paraíba. Aprovado pelo Comitê de Ética em Pesquisa em Seres Humanos do Hospital Universitário Lauro Wanderley sob o parecer 103/107, o estudo obedece aos critérios estabelecidos pela Resolução 196/96 do Conselho Nacional de Saúde.

\section{Resultados}

\subsection{Aplicabilidade da análise fatorial}

0 constructo, avaliado através do Alfa de Cronbach, indicou uma alta confiabilidade e a adequação da escala utilizada no instrumento de pesquisa (IEE), haja vista o resultado obtido $(\alpha=0,914)$. Além disso, a análise fatorial (AF) demonstrou ser aplicável sendo o valor do teste KMO igual a 0,881, o que representa um bom grau de ajuste ao uso do método. Aplicação da AF foi ratificada pelo teste de esfericidade de Bartlett, pois as variáveis deste estudo não são correlacionadas na população.

\subsection{Descrição da amostra}

0 questionário foi aplicado a 391 profissionais médicos, dos quais $220(56,3 \%)$ eram do sexo masculino e 171 (43,7\%) do sexo feminino. Os entrevistados encontram-se na faixa etária entre 23 e 45 anos, com idade média de 43,9. 0 tempo médio de formatura é de 20,06 anos e o tempo de trabalho médio é de 56,47 horas semanais. Os indivíduos entrevistados apresentam uma heterogeneidade evidenciada pelos altos valores do desvio padrão para o tempo de formado, a idade e para a carga horária semanal (Tabela 1).

\subsection{Extração dos fatores e cargas das variáveis}

0 número de fatores foi estimado através dos autovalores $(\geq 1)$ e da porcentagem da variância explicada ( $\geq 60 \%)$, que indicaram que as 44 variáveis não métricas do questionário podem ser explicadas por dez fatores. Com a aplicação da rotação Varimax, os fatores foram submetidos ao teste de confiabilidade Alfa de Cronbach, sendo dois deles com valores abaixo de 0,60 . As variáveis que faziam parte destes dois fatores foram desconsideradas e uma nova extração foi realizada, reduzindo o número de fatores para oito. 0 Apêndice 1 mostra as variáveis que formam cada fator, com suas respectivas cargas, bem como o valor da consistência de cada fator $(\alpha)$.

Com o intuito de facilitar a compreensão, a Figura 1 sumariza o resultado da $\mathrm{AF}$, mostrando o comportamento de cada fator com suas respectivas variáveis. Quanto mais próximo do centro está o fator, maior é o impacto de suas respectivas variáveis para a ocorrência de estresse em médicos.

\section{Discussão}

A análise do instrumento utilizado através do modelo Alfa de Cronbach demonstrou que a estrutura interna do instrumento (IEE) possui confiabilidade escalar. Através da AF foi possivel identificar oito fatores associados ao surgimento do estresse dentre os profissionais médicos da cidade de João Pessoa (PB). Alguns dos fatores evidenciados por essa ferramenta apresentaram confiabilidade com alto grau de importância, o que reforça a possível existência de variáveis que contribuem fortemente para o estresse no exercício da atividade médica. Dentre as variáveis que demonstram grande impacto estão: relacionamento com a equipe $(0,881)$, exercer atividades com instalações físicas

Tabela 1. Estatística descritiva.

\begin{tabular}{lcc}
\hline & Média & Desvio padrão \\
\hline Carga horária semanal & 56,47 & 21,569 \\
Tempo de formado & 20,06 & 11,186 \\
ldade & 43,90 & 11,513 \\
\hline
\end{tabular}




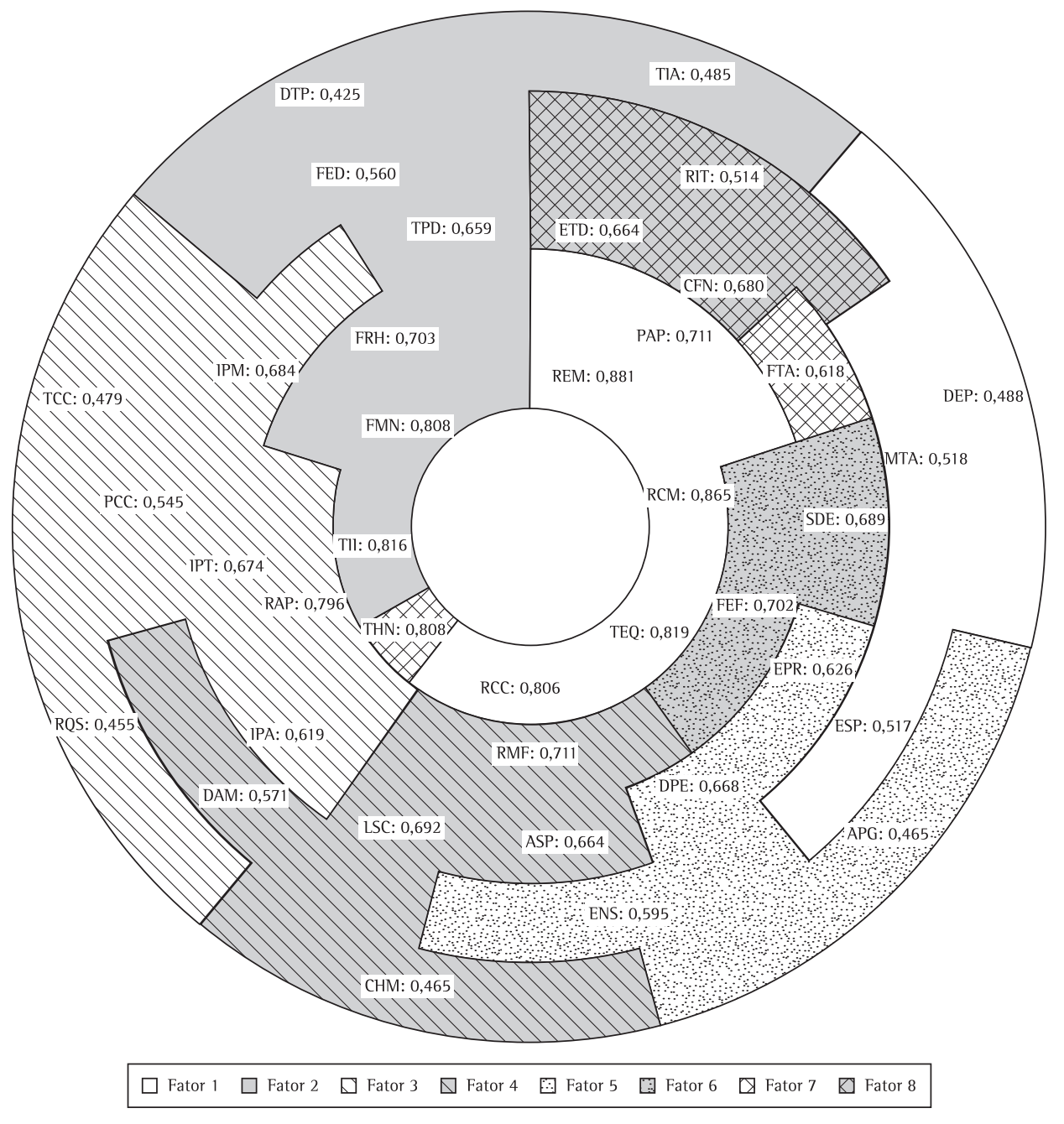

Figura 1. Relação das variáveis com o estresse.

inadequadas $(0,801)$, restrição da autonomia profissional $(0,796)$, exercer diversas funções no mesmo ambiente hospitalar (0,711), o esforço físico para cumprir a tarefa $(0,702)$ e trabalhar em horário noturno $(0,808)$.

Esses resultados corroboram o que existe na literatura sobre o assunto. Em uma pesquisa anterior realizada com o mesmo instrumento de coleta de dados com 117 médicos da rede hospitalar, nove fatores foram apontados como associados ao surgimento do estresse: relacionamento interpessoal e dedicação ao trabalho, organização do trabalho, maturidade profissional, estrutura, atendimento a pacientes, jornada de trabalho, emocional, qualidade na prestação do serviço e outros (SANTOS et al., 2008).

Cabana et al. (2007) relatam que os profissionais de um hospital público em Pernambuco apontaram como causas da sensação da sobrecarga do trabalho médico a carga horária diária/semanal de trabalho, bem como a privação de sono. Em outra pesquisa realizada em Goiás (SILVA, 2001), os médicos indicaram como fatores que contribuem para 0 desgaste no trabalho a jornada de trabalho, o desgaste com relação ao poder do médico, a remuneração, a diminuição do prestígio profissional e a piora das condições de trabalho como determinantes para essa sensação de desgaste entre os profissionais. De forma semelhante, na atual pesquisa, o trabalho em horário noturno e aspectos relacionados à autonomia do profissional apresentaram fortes impactos, como demonstrados pelas variáveis trabalhar em horário noturno $(0,808)$, restrição da autonomia $(0,796)$, indefinição do papel do médico $(0,684)$ e interferência de políticas institucionais no trabalho $(0,674)$. Como causas para mudanças em aspectos do exercício da profissão médica: a perda da autonomia, a remuneração, o estilo de vida, a 
saúde desse profissional, o comportamento ético e a relação médico-paciente. Nogueira-Martins (2003) aponta também o desenvolvimento de novos recursos terapêuticos e de diagnóstico, a influência da indústria farmacêutica e de equipamentos e as empresas compradoras de serviços médicos.

Conforme pode ser observado no Apêndice 1, o relacionamento com a equipe médica $(0,881)$, com os colegas médicos $(0,865)$, com a chefia $(0,806)$ e o trabalho em equipe $(0,819)$ são variáveis que contribuem significativamente para o estresse entre os médicos dessa amostra, tendo em vista os altos valores das cargas apresentados. Esse resultado levanta a hipótese da existência de problemas no relacionamento entre os médicos e demais profissionais. A alienação do trabalho médico é, segundo Silva (2001), oriunda do não reconhecimento pelo médico do resultado ou produto de seu trabalho devido à alta fragmentação nos processos de trabalho nos serviços de saúde, incluindo as diversas especialidades. Isso ocasiona a descaracterização e a desumanização do atendimento, de tal forma que o médico torna-se alienado e alienante nas relações interpessoais, assumindo uma postura acrítica e naturalizada do processo saúde-doença. Pode-se pensar, ainda, como causas dos problemas de relacionamento entre os médicos e os demais profissionais a alta carga de trabalho, determinada pela grande demanda do sistema de saúde, e o vínculo empregatício em diversas instituições, que interfere na solidificação do relacionamento com os colegas.

Oliveira e Kilimnik (2007), em um estudo com médicos ultrassonografistas, identificaram que o fator mais citado como fonte de tensão no trabalho foi o inter-relacionamento, seguido por falta de possibilidade de crescimento, carga de trabalho, responsabilidade individual, papel gerencial do indivíduo, equilíbrio entre a vida pessoal e profissional e clima organizacional. Todavia, a necessidade de atualização, através de educação continuada e participação em cursos e eventos científicos da área, geralmente não representa fonte de tensão para esses profissionais, fato que foi ratificado pelo nosso estudo, onde a variável manter-se atualizado apresentou baixo impacto no primeiro fator $(0,518)$. Outros fatores mencionados pelos médicos na pesquisa de Oliveira e Kilimnik (2007) incluíram a estrutura da organização, as relações de poder e política, a baixa tecnologia dos aparelhos de ultrassonografia, a relação entre o médico ultrassonografista, o solicitante e o paciente, a necessidade de vários empregos para a subsistência e os fatores ergonômicos.
Observando a rotina de profissionais de saúde em um setor de emergência, Boller (2003) identificou que grande parte dos fatores causadores de estresse era proveniente do ambiente de trabalho. Algumas variáveis identificadas incluem, em ordem decrescente, sobrecarga e pressão, tipo de paciente atendido, deficiência de pessoal, falta de resolução no trabalho, problemas na organização, realização de tarefas sem bom acabamento e precisão, conflitos entre profissionais atuantes na unidade, acomodações inapropriadas e falta de material. As últimas variáveis (falta de material e acomodações), todavia, neste estudo apresentaram importância contrária aos resultados de Boller (2003), com altos impactos para o estresse dos médicos em João Pessoa (PB), com carga fatorial de 0,808 para a falta de material e 0,816 em trabalhar em instalações físicas inadequadas.

Em um estudo realizado em Passo Fundo (RS) no centro cirúrgico de um hospital geral, com 29 profissionais da área de saúde, os estressores referidos pela equipe foram classificados em: decorrentes da relação entre anestesistas, cirurgiões e enfermagem; da falta e/ou insuficiência de materiais e equipamentos; da insuficiência e/ou falta de pessoal, aliada a aspectos administrativogerenciais e qualificação da equipe; da demanda de procedimentos cirúrgicos e estressores relacionados à condição do paciente no centro cirúrgico (STUMM et al., 2008).

Observou-se neste estudo que "prestar assistência ao paciente", independente de seu quadro clínico, também colabora significativamente para o estresse dos médicos. Segundo Boller (2003), o estresse gerado pode ser decorrente da alta demanda de pacientes que procuram os serviços de saúde em busca do seu direito à saúde. E da mudança na concepção da relação médico-paciente, que se encontra em processo de humanização, buscando sensibilizar os médicos diante do sofrimento da doença (CAPRARA; RODRIGUES, 2004). Espera-se que eles sejam atenciosos, dedicados e empáticos com seus pacientes.

\section{Conclusão}

A presente pesquisa detectou oito fatores associados para o surgimento de estresse em médicos que exerciam suas atividades em hospitais na cidade de João Pessoa (PB). É possível apontar variáveis que possuem maior impacto dentro dos fatores. Podem-se citar: relacionamento com a equipe médica, relacionamento com os colegas médicos, trabalhar em equipe, relacionamento com a chefia, trabalhar em instalações físicas inadequadas, falta de material necessário ao trabalho e trabalhar em horário noturno. 
0 conhecimento dessas informações é de fundamental importância, podendo subsidiar tomadas de decisão para mitigar os efeitos do estresse nesses profissionais, garantindo-lhes as condições necessárias para realizarem seu trabalho adequadamente. Além disso, visto que o estresse em médicos pode interferir na sua produtividade, na qualidade da prestação do serviço e, consequentemente, na satisfação do usuário, a gestão do estresse nesses profissionais é de suma relevância. Os dados gerados neste estudo auxiliam na priorização de ações considerando-se o impacto das variáveis para o estresse em médicos nos hospitais.

É importante salientar que o estudo apresenta algumas limitações. A primeira condiz ao número de médicos utilizados na amostra, que não é representativo para a população dessa categoria na cidade. A outra está relacionada ao instrumento utilizado, que inicialmente foi proposto para profissionais de enfermagem e não para médicos. Apesar de muitas variáveis terem sido usadas sem alterações e outras repensadas e/ou reformuladas no sentido de promover melhor interface com o trabalho médico, o estresse em médicos tem suas particularidades e deve ser estudado por um instrumento específico.

\section{Referências}

BALLONE, G. J. Estresse: curso de psicopatologia. 2002. Disponível em: <www.psiqweb.med.br/cursos/stress 1.html>. Acesso em: 13 maio 2002.

BAUK, D. A. Stress. Revista Brasileira de Saúde Ocupacional, v. 13, n. 50, p. 28-36, 1985.

BOLLER, E. Estresse no setor de emergência: possibilidades e limites de novas estratégias gerenciais. Revista Gaúcha de Enfermagem, v. 24, n. 3, p. 336-45, 2003.

CABANA, M. C. F. L. et al. Transtornos mentais comuns em médicos e seu cotidiano de trabalho. Jornal Brasileiro de Psiquiatria, v. 56, n. 1, p. 33-40, 2007.

CAMELO, S. H. C.; ANGERAMl, E. L. S. Sintomas de estresse nos trabalhadores atuantes em cinco núcleos de saúde da família. Revista. Latino-Americana de Enfermagem, v. 12, n. 1, p. 14-21, 2004.

CANDEIAS, N. M. F.; ABUJAMRA, A. M. D.; LIM, T. A. "Stress" em um instituto de cardiologia da cidade de São Paulo. Revista Brasileira de Saúde Ocupacional, v. 16, n. 64, 1988.

CAPRARA, A.; RODRIGUES, J. A relação assimétrica médicopaciente: repensando o vínculo terapêutico. Ciência \& Saúde Coletiva, v. 9, n. 1, p. 139-146, 2004.

CORRAR, L. J.; PAULO, E.; DIAS FILHO, J. M. Análise multivariada: para os cursos de administração, ciências contábeis e economia. São Paulo: Atlas, 2007.

DEJOURS, C. A loucura do trabalho. 5 ed. São Paulo: Cortez Oboré, 1992.

FRANÇA, A. C. L.; RODRIGUES, A. L. Stress e Trabalho: uma abordagem psicossomática. 2. ed. São Paulo: Atlas, 1999.

FRASQUILHO, M. A. Medicina, médicos e pessoas: compreender o stresse para prevenir o burnout. Acta Médica Portuguesa, v. 18, p. 433-444, 2005.

HAIR Jr., J. F. et al. Análise multivariada de dados. 5. ed. Porto Alegre: Bookman, 2005.
HAWTON, K. et al. Suicide in doctors: a study of risk according to gender, seniority and specialty in medical practitioners in England and Wales, 1979-1995. Journal of Epidemiology and Community Health, v. 55, p. 296-300, 2001.

IVERSEN, A.; RUSHFORTH, B.; FORREST, K. How to handle stress and look after your mental health. British Medical Journal, v. 338, p. 1139-1142, 2009.

LENTINE, E. C.; SONODA, T. K.; BIAZIN, D. T. Estresse de profissionais de saúde das unidades básicas do município de Londrina. Terra e Cultura, v. 19, n. 37, 2003.

LIMONGI-FRANÇA, A. C. Psicologia do trabalho: psicossomática, valores e práticas organizacionais. São Paulo: Saraiva, 2008.

LOURENÇO, E. A. Estresse profissional no exercício da medicina - origem, fatores mantenedores, conseqüências e soluções. Revista Perspectivas Médicas, v. 9, p. 7-8, 1998.

MELEIRO, A. M. A. S. Suicídio entre médicos e estudantes de medicina. Revista da Associação Médica Brasileira, v. 44, n. 2, p. 135-40, 1998.

MENDES, R. Patologia do trabalho. Rio de Janeiro: Atheneu, 1995.

NASCIMENTO SOBRINHO, C. L. et al. Condições de trabalho e saúde dos médicos em Salvador, Brasil. Revista da Associação Médica Brasileira, v. 52, n. 2, p. 97-102, 2006.

NOGUEIRA-MARTINS, L. A. Atividade Médica: fatores de risco para a saúde mental do médico. Revista Brasileira de Clínica e Terapêutica, v. 20, p. 355-64, 1991.

NOGUEIRA-MARTINS, L. A. Saúde mental dos profissionais de saúde. Revista Brasileira de Medicina do Trabalho, v. 1, n. 1, p. 56-68, 2003.

OLIVEIRA, F. A.; KILIMNIK, Z. M. Avaliação dos fatores de pressão no trabalho médico e sua relação com o estresse: um estudo em uma unidade de ultra-sonografia da rede pública em comparação com unidades da rede privada. Revista de Administração da FEAD - Minas, v. 4, n. 1, p. 105-24, 2007.

PACHECO Jr., W. et al. Gestão da segurança e higiene do trabalho: contexto estratégico, análise ambiental, controle e avaliação das estratégias. São Paulo: Atlas, 2000.

RAMOS-CERQUEIRA, A. T. A.; LIMA, M. C. P. A formação da identidade do médico: implicações para o ensino de graduação em Medicina. Interface - Comunicação, Saúde, Educação, v. 6, n. 11, p. 107-16. 2002.

RODRIGUES, A. L. $O$ "stress" no exercício profissional da Medicina - uma abordagem psicossocial. 1998. Tese (Doutorado em Psicologia) - Programa de Psicologia Social, Pontifícia Universidade Católica de São Paulo, São Paulo, 1998.

SANTOS, C. L. M. et al. Estresse em médicos: um estudo num hospital público de João Pessoa - Brasil. In: AREZES, P. et al. Segurança e Higiene Ocupacionais - SHO 2008. Guimarães: Sociedade Portuguesa de Segurança e Higiene Ocupacionais, 2008. p. 287-290.

SCHERNHAMMER, E. S.; COLDITZ, G. A. Suicide Rates Among Physicians: A Quantitative and Gender Assessment (Meta-Analysis). The American Journal of Psychiatry, v. 161, n. 12, p. 2295-2302, 2004.

SILVA, M. M. A. Trabalho médico e o desgaste profissional: pensando um método de investigação. 2001. $186 \mathrm{f}$. Dissertação (Mestrado em Saúde Coletiva) - Faculdade de Ciências Médicas, Universidade Estadual de Campinas, Campinas, 2001.

SPIELBERGER, C. D. Anxiety as an emotional state. In: SPIELBERGER, C. D. Anxiety. currents trends in theory and research. New York: Academic Press, 1972. p. 109-48.

STACCIARINI, J. M. R.; TROCOLI, B. T. Instrumento para mensurar o estresse ocupacional: inventário de estresse em enfermeiros (IEE). Revista Latino-Americana de Enfermagem, v. 8, n. 6, p. 40-9, 2000.

STUMM, E. M. F. et al. Estressores e sintomas de estresse vivenciados por profissionais em um centro cirúrgico. Revista Mineira de Enfermagem, v. 1, n. 12, p. 54-63, 2008. 


\section{Stress factors in physicians' activity in João Pessoa (Brazil)}

\section{Abstract}

The objective of this study is to verify what factors impact directly on the development of stress among physicians in João Pessoa (PB) based on the variables of an adaptation made to the Stress in Nurses lnventory (IEE) proposed by Stacciarini and Trocoli (2000). The data were collected through a semi-structured questionnaire composed of identification questions and multiple-choice questions (44) applied to 391 hospital physicians. The non-metric variables were submitted to Factor Analysis, which indicated eight determinant factors for stress among these professionals. It is possible to highlight variables that cause greater impact, such as: "medic-staff relationships", "colleague relationships", "group-working”, "relationships with the administration”, "working in inadequate environments", "lack of necessary equipment" and "working at night". These results may contribute to stress management among physicians.

\section{Keywords}

Stress. Physicians. Hospitals.

Apêndice 1. Composição dos fatores com respectivas cargas e valores de consistência $(\varepsilon \pm)$.

\begin{tabular}{|c|c|c|c|}
\hline Fator & Variáveis & Carga & $\alpha$ \\
\hline \multirow{8}{*}{1} & Relacionamento com a equipe médica (REM) & 0,881 & \multirow{8}{*}{0,886} \\
\hline & Relacionamento com os colegas médicos (RCM) & 0,865 & \\
\hline & Trabalhar em equipe (TEQ) & 0,819 & \\
\hline & Relacionamento com a chefia (RCC) & 0,806 & \\
\hline & Prestar assistência ao paciente (PAP) & 0,711 & \\
\hline & \begin{tabular}{|l|} 
Manter-se atualizado (MTA) \\
\end{tabular} & 0,518 & \\
\hline & A especialidade em que trabalha (ESP) & 0,517 & \\
\hline & Dedicação exclusiva à profissão (DEP) & 0,488 & \\
\hline \multirow{7}{*}{2} & Trabalhar em instalações físicas inadequadas (T11) & 0,816 & \multirow{7}{*}{0,822} \\
\hline & Falta de material necessário ao trabalho (FMN) & 0,808 & \\
\hline & Falta de recursos humanos (FRH) & 0,703 & \\
\hline & \begin{tabular}{|l|} 
Trabalhar com pessoas despreparadas (TPD) \\
\end{tabular} & 0,659 & \\
\hline & Falta de espaço no trabalho para discutir as experiências, tanto as positivas como as negativas (FED) & 0,560 & \\
\hline & \begin{tabular}{|l|l|} 
Trabalhar em ambiente insalubre (TIA) \\
\end{tabular} & 0,485 & \\
\hline & Distanciamento entre a teoria e a prática (DTP) & 0,425 & \\
\hline \multirow{7}{*}{3} & Restrição da autonomia profissional (RAP) & 0,796 & \multirow{7}{*}{0,816} \\
\hline & Indefinição do papel do médico (IPM) & 0,684 & \\
\hline & Interferência da política institucional no trabalho (IPT) & 0,674 & \\
\hline & Impossibilidade de prestar assistência direta ao paciente (IPA) & 0,619 & \\
\hline & \begin{tabular}{|l|} 
Ter um prazo curto para cumprir ordens (PCC) \\
\end{tabular} & 0,545 & \\
\hline & Trabalhar em clima de competitividade (TCC) & 0,479 & \\
\hline & Responsabilizar-se pela qualidade de serviço que a instituição presta (RQS) & 0,455 & \\
\hline \multirow{5}{*}{4} & Responder por mais de uma função neste emprego (RMF) & 0,711 & \multirow{5}{*}{0,747} \\
\hline & Levar serviço para fazer em casa (LSC) & 0,692 & \\
\hline & Administrar ou supervisionar o trabalho de outras pessoas (ASP) & 0,664 & \\
\hline & \begin{tabular}{|l|l|l|} 
Desenvolver atividades além da minha função ocupacional (DAM) \\
\end{tabular} & 0,571 & \\
\hline & \begin{tabular}{|l} 
Cumprir na prática uma carga horária maior (CHM) \\
\end{tabular} & 0,465 & \\
\hline \multirow{4}{*}{5} & Desenvolver pesquisa (DPE) & 0,668 & \multirow{4}{*}{0,710} \\
\hline & \begin{tabular}{|l|} 
Executar procedimentos rápidos (EPR) \\
\end{tabular} & 0,626 & \\
\hline & Ensinar o aluno (ENS) & 0,595 & \\
\hline & Prestar assistência a pacientes graves (APG) & 0,465 & \\
\hline \multirow{2}{*}{6} & Fazer esforço físico para cumprir o trabalho (FEF) & 0,702 & \multirow{2}{*}{0,645} \\
\hline & \begin{tabular}{|l} 
Sentir desgaste emocional com o trabalho (SDE) \\
\end{tabular} & 0,689 & \\
\hline \multirow{2}{*}{7} & Trabalhar em horário noturno (THN) & 0,808 & \multirow{2}{*}{0,611} \\
\hline & \begin{tabular}{|l|} 
Fazer turnos alternados de trabalho (FTA) \\
\end{tabular} & 0,618 & \\
\hline \multirow{3}{*}{8} & Começar em uma função nova (CFN) & 0,680 & \multirow{3}{*}{0,606} \\
\hline & \begin{tabular}{|l|} 
Executar tarefas distintas simultaneamente (ETD) \\
\end{tabular} & 0,664 & \\
\hline & Resolver imprevistos que acontecem no local de trabalho (RIT) & 0,514 & \\
\hline
\end{tabular}

Composição dos fatores com respectivas cargas. 